\title{
Fighting Corona Virus Using Convalescent Plasma as a Potential Therapy: A Review
}

\author{
Umesh $\mathrm{Y}^{1}$, Prashant Kumar $\mathrm{S}^{1 *}$, Raj $\mathrm{K}^{2}$ and Shah $\mathrm{B}^{3}$ \\ ${ }^{1}$ Department of Pharmacy Practice, East west College of Pharmacy, India \\ ${ }^{2}$ Neuroscience Division, Department of Pharmacology, ISF College of Pharmacy, India \\ ${ }^{3}$ Department of Pharmacology, ISF College of Pharmacy, India
}

*Corresponding author: Prashant Kumar Sah, Pharm D Scholar, Department of Pharmacy

Practice, East West College of Pharmacy Bengaluru, Karnataka-560091, India, Tel: +919206436315; Email: prashantsah65933@ gmail.com

\section{Abstract}

With the irruption of unknown respiratory disease in Wuhan, China, in December 2019, a novel corona virus, Severe Acute Respiratory Syndrome Corona Virus-2 (SARS-CoV-2), aroused the eye of the whole world and therefore the World Health Organization declared SARS-CoV-2 as public health emergency of international concern in China. Corona virus COVID-19 has affected 213 countries and territories around the world and 2 international conveyances as of June 1, 2020. 62,88,301 cases, with $3,74,327$ deaths has been reported as of June 1,2020. Currently, no specific vaccine, antivirals or monoclonal antibodies available for COVID-19, although some drugs are in rapid development and may be available in a short time. Human convalescent serum is an alternative option for COVID-19 disease prevention and treatment that could be available as sufficient numbers of people $(28,58,645)$ have recovered and are eligible to donate serum containing immunoglobulins. Administration of antibodies to the susceptible person against an exposed agent for the prevention or treatment an infectious disease because of that exposed agent is called Passive antibody therapy. Thus, passive antibody administration is the only means of providing immediate immunity to susceptible persons. This review focused on Convalescent Plasma which reduces mortality and appears to be safe. So, we suggest a well designed clinical trial should be conducted in this context.

Keywords: COVID-19; Corona virus; Passive antibody therapy; Convalescent plasma

Abbreviations: SARS-CoV-2: Severe Acute Respiratory Syndrome Corona Virus-2; WHO: World Health Organization; SARS-CoV-1: Severe Acute Respiratory Syndrome Corona Virus-1; MERS-CoV-1: Middle East Respiratory Syndrome Corona Virus-1; H1N1: Influenza A virus; CP: Convalescent Plasma; CT: Computer-assisted Tomography; IQR: Interquartile Range.

\section{Introduction}

A group of unrevealed pneumonia patients was found in December 2019 in Wuhan, China, caused by a new corona virus (nCoV), which was provisionally titled as 2019 novel corona virus (2019-nCoV) by the WHO on 7th January 2020 [1]. The virus was later renamed as Severe Acute Respiratory Syndrome corona virus 2 (SARS-CoV-2), and the disease which it seeds was called as corona virus Disease 2019 (COVID-19). Corona virus COVID-19 has affected 213 countries and territories around the world and 2 international conveyances as of June 1, 2020. 62,88,301 cases, with 3,74,327 deaths has been reported as of June 1, 2020 [2]. No specific antiviral agents targeting the novel virus has been approved until now, while some drugs under investigation, such as remdesivir and lopinavir/ritonavir $[3,4]$. The management of lung injury due to COVID-19 is controversial, because clearance of viral infection is late and has complications [5- 


\section{International Journal of Biochemistry \& Physiology}

7]. The vaccine and antiviral drugs are not available to treat of COVID-19 therefore it is crucial to develop strategy for COVID-19 management. The patients are managed mainly by providing supportive treatments. Combination of lowdose systematic corticosteroids, anti-viral and atomization inhalation of interferon has been advised as therapeutic option for management of severely ill COVID-19 patients [8]. The immune system makes antibodies, (proteins that attach to virus and hinder the infection) to fight with viruses. When a person's body develops antibodies responding to infection or on vaccine administration, it's called active immunity. It takes about 7-14 days for initial increase in development of active immunity. Active immunity against some viruses and vaccines may last decades or even lifelong. Convalescent plasma (CP) also called passive immunotherapy is chosen if particular vaccines or drugs are not available for emerging infection and disease related. Arabi et al tested the feasibility, efficacy and safety of convalescent plasma therapy clinically in critically ill MERS patients and found that convalescent plasma do have immunotherapeutic strength for managing MERS-CoV infection. Additionally, it's been reported that CP collected from patients recovered from SARS is effective for managing other SARS patients. World Health Organization (WHO) under Blood Regulators Network suggested the use of $\mathrm{CP}$ or serum when vaccines and antiviral drugs were unavailable for an emerging virus. CP can be collected from COVID-19 patients who had recovered and had gained humeral immunity against the virus. CP contains huge amount of Neutralizing antibodies which can neutralize SARS-CoV-2 and eradicate the virus from blood and pulmonary tissues [9].

Many research papers published in the last few months and the record setting leaps in vaccine development have not been fast enough to keep up with the blistering speed of the ongoing pandemic. This visualization contains that $\mathrm{CP}$ is an alternative for the prophylaxis and therapy of COVID-19 infection which could be readily available when ample numbers of peoples recovered are present and can donate serum that contains immunoglobulin. This review will highlights the key role of convalescent plasma as a potential therapy in finding therapeutic options for novel virus and therefore counters its damaging effects.

\section{Diagnostic Techniques of Covid-19}

\section{Reverse- Transcription Polymerase Chain Reaction (RT- PCR)}

Polymerase Chain Reaction (PCR) is very common technique and is now used to detect SARS- CoV-2 for existence of viral RNA. In RT-PCR, sample from nose or throat is collected as swab and proteins, fats and other molecules are removed from sample to leave only RNA using reagents. Sample is then kept in test kit, and the enzymes present in the kit copy RNA into DNA, which is then enlarged which allows detection of virus by PCR machine which cycles the temperature so that around 35 billion viral DNA copies are made from every original single viral RNA strand. Fluorescent markers are used which binds the amplified DNA and produces light, which is read by machine to produce result. The result is decided based on threshold of the intensity of light.

\section{Loop-Mediated Amplification (Lamp)}

LAMP is similar in process to RT-PCR but a constant temperature of 60-65 degree Celsius is required instead using temperature changes series for producing viral RNA copies. In LAMP, sample is collected using swab from throat or nose, the viral RNA present in the sample is transformed to DNA for copying. The viral DNA is then amplified by LAMP technique and reagents are then detected when the reaction mixture becomes cloudy attributable to production of chemical called magnesium phosphate. The cloudiness can be seen by naked eyes, so allows easy diagnosis of COVID-19.

\section{Lateral Flow (Colloidal Immune Chromatography)}

This test is used to detect antibodies to disease and antigen in patient blood. In this technique, a drop of patient's blood is collected either from vein or small prick and kept on a spongy pad present in the test kit. Few drops of diluting fluid called buffer are the added to help blood sample to move across the kit. As blood moves through device, antibodies anti to SARS-CoV-2 if present in sample gets attached to reagents in the device, arresting antibodies on the test and control lines. This capturing and binding process results in color change along the test and control lines visible by eye producing one, two or three lines which depends on the antibody type present (IgM or IgG).

\section{Enzyme Linked Immune Sorbent Assay (ELISA)}

This test uses enzyme linked to antibodies that can attach the molecule that is being tested for and cause color change that is measured by specialized mechanism. The strength of the colour change decides the result. This test can both detect antigen and antibody in blood sample [10].

\section{Passive Antibody Therapy}

Passive antibody therapy refers to administering antibodies against pathogen to exposed individual for the prophylaxis and treatment of disease due to that pathogen. Active vaccination needs the initiation of immune response which requires time for developing and differs based on recipient of the vaccine. Thus, administering passive antibody is last option for provision of immediate immunity to exposed persons. The history of passive antibody treatment goes back to the 1890s, when the only means for curing certain diseases caused by infections before the antimicrobial 


\section{International Journal of Biochemistry \& Physiology}

therapy in the 1940s developed [11,12]. Experience from previous epidemics with other corona viruses, such as SARSCoV1, reveals that convalescent sera possess neutralizing antibodies to the relevant virus [13]. In SARS-CoV-2 case, the passive antibody treatment would arbitrate protection by viral neutralization. Other possible mechanisms for protection may be phagocytosis or antibody dependent cellular cytotoxicity. Human convalescent sera collected from person who has been cured from COVID-19, mAbs, or preparations made in some animal hosts like genetically engineered cows which generate human antibody are the probable source of antibody for SARS-CoV-2 [14]. Various preparations will be under development soon, but for instant use, antibody found in human convalescent sera is only available presently. The donors for $\mathrm{CP}$ will increase as more peoples get infected with COVID-19 and recover. Passive antibody treatment is more effective if used for prophylaxis rather than management of disease. When considered of management, antibody will be most effective if given soon after the symptoms start. The variation in effectiveness is seen and reason is not understood but can be considered that passive antibody exerts its effect by counteracting the initial inoculums, which will be much smaller than established disease [15]. Another explanation is that antibody exerts its effect by modifying the inflammatory response, and is easily achieved during the initial immune response, a stage that may be asymptomatic [16]. For example, in pneumococcal pneumonia, passive antibody therapy showed high effectiveness when given soon after the symptoms onset, and when delayed past the third day of disease, benefit was not observed [17]. The antibody should be administered in sufficient amount to be effective; antibody administered to an exposed person, circulates in the blood, reaches tissues, and provides protection against infection. The protection can be effective for weeks to months and depends on composition and amount of immunoglobulin administered [18].

\section{Historical Experience of Convalescent Plasma Use against Corona Virus Diseases}

Two other epidemics with corona viruses have been seen in the twenty-first century associated with high mortality, SARS1 in 2003 and MERS in 2012. MERS became endemic in the Middle East and activated a secondary crucial outbreak in South Korea but SARS1 epidemic was suppressed. The absence of specific treatment and high death rate in both outbreaks led to convalescent serum use. One study involved 80 SARS patients for treatment in Hong Kong. Before day 14, the patients being treated showed improved prognosis as they were discharged from hospital before day 22 , and consistency with the notion was seen that earlier administration is most effective. Additionally, improved prognosis was seen at the time of treatment in PCR positive patients but negative serologically for corona virus [19]. Some unreliable information on the convalescent serum use in critically ill persons are also available. In Taiwan, 3 SARS patients were administered with $500 \mathrm{~mL}$ convalescent serum which resulted in alleviation in serum virus titer, and all 3 survived [20]. In South Korea, three MERS patients were managed using convalescent serum, and only 2 patients developed neutralizing antibody in their serum [21]. The latter study highlighted a question in using convalescent sera that some who get cured from viral disease may not have high titers of neutralizing antibody [22]. Consistent with this point, convalescent sera of 99 SARS patients were analyzed and it was revealed that neutralizing antibody was developed in 87 patients, with a geometric mean titer of 1:61 which suggested that decline in antibody is seen with time and/or few patients produces high-titer responses [23-25].

\section{Effectiveness of Convalescent Plasma therapy with COVID-19}

The pilot study conducted by the Kai Duan et al at different hospitals of China from January 23 to 19th February, 2020, involved ten critical COVID-19 patients to evaluate the effectiveness of therapy with CP [26]. 10 severe COVID-19 patients (6 males and 4 females) were involved and were transfused with CP. The average age was 52.5 years and the average time between symptoms onset to hospital admission and transfusion of CP was 6 days. Fever, cough and breathlessness are mostly seen symptoms at disease onset. When effectiveness of CP treatment in COVID-19 was explored, single dose of 200-mL CP administration was tolerated in well manner, and significant improvement were seen with the rise in saturation of oxyhemoglobin in 3 days, accompanied by neutralization of viraemia rapidly. After the transfusion, every patient had serum SARS-CoV-2 RNA negativity, raised oxygen saturation, lymphocyte counts, and improved liver function and C-Reactive Proteins. This study revealed reduction in inflammation and exaggeration of the immune system by antibodies present in CP and mortality rates were $0 \%(0 / 10)$. Major adverse effects were not observed and no plasma related risk like pulmonary injury, antibody dependent infection and transfusion related acute injury was seen. Therefore, treatment with CP showed potential therapeutic effect and had low risk in treating severely ill COVID-19 patients. A dose of CP containing high amount of neutralizing antibodies can alleviate the viral load rapidly and improves clinical outcomes [25]. We also evaluated the study conducted by Shen et al in which involved five critical COVID-19 were treated using CP from recovered individuals in the Shenzhen Third People's Hospital, China. All 5 patients had severe respiratory failure and were on mechanical ventilation. Four patients without any other diseases were administered with $\mathrm{CP}$ around 20th 


\section{International Journal of Biochemistry \& Physiology}

day of hospitalization and patient who had hypertension and mitral valve insufficiency were transfused on 10th day. IgG and IgM anti-SARS-CoV-19 antibodies were demonstrable in the donor plasma which neutralized the virus when in vitro culture study was conducted. While these patients were also continuously receiving lopinavir/ritonavir and interferon as antiviral therapy, CP use might have contributed to their recovery as health status of all patients improved after 1 week of transfusion, as body temperature was normalized as well as Sequential Organ Failure Assessment scores and PA02/FIO2 ratio were improved evidencing the above statement. In addition, increase in the patients neutralizing antibody titers was observed and respiratory samples were tested negative for SARS-CoV-2 between 1 and 12 days after transfusion [27].

\section{How Convalescent Therapy Works?}

One of the hopeful treatments that have emerged is CP, or immune plasma. The CP therapy uses antibodies obtained from recovered COVID-19 patients. These antibodies develop as body's natural immune response infective agents (e.g. novel corona virus). These antibodies possess high specificity to the infective agents and eradicates new corona virus from the body of patients [27]. Once the patient recovers, the blood is obtained so that antibodies present can be used for therapy of other infected patients as shown in fig.1. Once proven safe, obtained blood is processed to extract plasma which contains antibodies. The plasma rich in antibody, after extraction is then transfused into infected patients as a post-exposure prophylaxis. Antibodies derived from CP may neutralize a virus by inhibiting replication or by binding without interfering with viral replication $[27,28]$. A patient treatment with infectious disease generates antibodies that might protect against later infections with the same microorganism. This acquired immunity when it can be prevented from the infection by giving serum to those at risk of infection. CP, a post-infection treatment, has had shown limited and moderate success in China, previously for MERS and SARS-1 for COVID-19, and could serve as a short-term solution to suppress global mortality rates. As the number of infections of COVID increases, the CP of infected patients may be donated or harvested for concurrent treatment or future use until an effective antibody is found [27].

\section{Risks and ethical considerations of CP therapy}

Human immunoglobulin therapy has been associated with significant raise same-day thrombotic event risk $(0.04$ to $14.9 \%$ ) [29] which indicates the need for studying the efficacy of early intervention treatment with CP or SARSCoV-2-specific hyperimmune globulin in acute respiratory disease patients in this outbreak. The basic biological information of SARS-CoV-2 may better reflect the circulating virus in the population and could be a valid treatment option.

Lack of high quality studies and proper donor selection are other issues that must be considered. The production and the use of convalescent plasma must be carried out based on precise ethical and controlled [30,31].

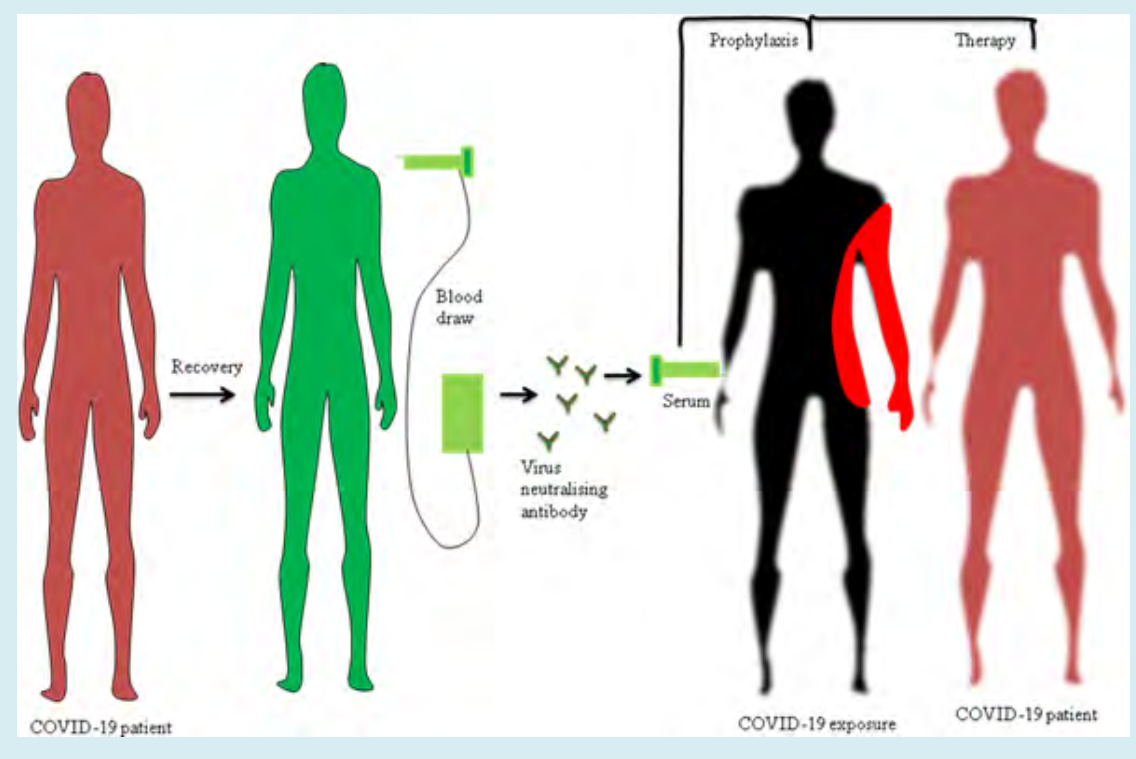

Figure 1: Schematic representation of the use of convalescent sera for treatment of Corona virus. 


\section{International Journal of Biochemistry \& Physiology}

\section{Conclusion}

The epidemic of severe acute respiratory syndrome corona virus 2 (SARS-CoV-2) originating in Wuhan, China, has rapidly spread worldwide currently, antiviral agents specific to novel virus has not been approved whereas drugs like remdesivir and lopinavir/ritonavir are under investigation. Therefore the use of passive antibody therapy for COVID-19 makes sense, as it's available immediately and can be tried on people. Evidence shows that CP therapy possesses a significant therapeutic effect and is associated with lower risk in the treating critical COVID-19 patients. Single dose of $\mathrm{CP}$ with adequate amount of neutralizing antibodies reduces the viral load rapidly and improves clinical outcomes. Therefore, considering the possible seriousness and high fatality rate of SARS-CoV-2, its necessary to provide as many people as possible a chance to get safe and effective products that could save their lives. It will be worthy to study the safety and efficacy of CP transfusion in SARS-CoV-2-infected patients with the context of a well designed clinical trial and efforts should be given to not only feasibility of the CP therapy but also on facilitating access to widespread and affordable treatments, especially in developing countries.

\section{References}

1. Huang C, Wang Y, Li X, Ren L, Zhao J, et al. (2020) Clinical features of patients infected with 2019 novel corona virus in Wuhan. China Lancet 395(10223): 497-506.

2. H Lu (2020) Drug treatment options for the 2019-new corona virus (2019-nCoV). Biosci Trends 14(1): 69-71.

3. Wang M, Cao R, Zhang L, Yang X, Liu J (2020) Remdesivir and chloroquine effectively inhibit the recently emerged novel corona virus (2019-nCoV) in vitro. Cell Res 30: 269-271.

4. Russell CD, Millar JE, Baillie JK (2020) Clinical evidence does not support corticosteroid treatment for 2019nCoV lung injury. Lancet 395: 473-475.

5. Shang L, Zhao J, Hu Y, Du R, Cao R (2020) On the use of corticosteroids for 2019-nCoV pneumonia. Lancet 395: 683-684.

6. Aimme Cunningham, (2020) plasma from recovered COVID-19 patients treat the sick?. ScienceNews april 3.

7. Liu Y, Li J, Feng Y (2020) Critical care response to a hospital outbreak of the 2019- nCoV infection in Shenzhen, China. Critical Care 56.

8. Marano G(2016) Convalescent plasma: New evidence for an old therapeutic tool? Blood Transfus 14(2): 152-157.
9. Casadevall A, Scharff MD (1995) Return to the past: the case for antibody-based therapies in infectious diseases. Clin Infect Dis 21(1): 150-161.

10. What tests could potentially be used for the screening, diagnosis and monitoring of COVID-19 and what are their advantages and disadvantages?

11. Casadevall A, Dadachova E, Pirofski LA (2004) Passive antibody therapy for infectious diseases. Nat Rev Microbiol 2(9): 695-703.

12. Zhang JS (2005) A serological survey on neutralizing antibody titer of SARS convalescent sera. J Med Virol 77(2): 147-150.

13. Beigel JH (2018) Safety and tolerability of a novel, polyclonal human anti-MERS corona virus antibody produced from transchromosomic cattle. a phase 1 randomised, double- blind, single-dose-escalation study. Lancet Infect Dis 18(4): 410-418.

14. Robbins JB, Schneerson R, Szu SC (1995) Perspective: hypothesis: serum IgG antibody is sufficient to confer protection against infectious diseases by inactivating the inoculum. J Infect Dis 171(6): 1387-1398.

15. Casadevall A, Pirofski LA (2003) Antibody-mediated regulation of cellular immunity and the inflammatory response. Trends Immunol 24(9): 474-478.

16. Casadevall A, Scharff MD (1994) Serum therapy revisted: animal models of infection and development of passive antibody therapy. Antimicrob Agents Chemother 38(8): 1695-1702.

17. Casadevall A, Pirofski L (2020) The convalescent sera option for containing COVID-19, J Clin Invest 130(4): 1545-1548.

18. Cheng Y (2005) Use of convalescent plasma therapy in SARS patients in Hong Kong. Eur J Clin Microbiol Infect Dis 24(1): 44-46.

19. Yeh KM (2005) Experience of using convalescent plasma for severe acute respiratory syndrome among healthcare workers in a Taiwan hospital. J Antimicrob Chemother 56(5): 919-922.

20. Arabi YM (2016) feasibility of using convalescent plasma immunotherapy for MERS- CoV infection, Saudi Arabia. Emerging Infect Dis 22(9): 1554-1561.

21. van Erp EA, Luytjes W, Ferwerda G, van Kasteren PB (2019) Fc-mediated antibody effector functions during respiratory syncytial virus infection and disease. Front Immunol 10: 548. 


\section{International Journal of Biochemistry \& Physiology}

22. Jenks JA, Goodwin ML, Permar SR (2019) The roles of host and viral antibody fc receptors in herpes simplex virus (HSV) and human cytomegalovirus (HCMV) infections and immunity. Front Immunol 10: 2110.

23. Kai Duana, Bende Liuc, Cesheng Lid, Huajun Zhange, Ting Yuf, Jieming Qugh, et al. Effectiveness of convalescent plasma therapy in severe COVID-19 patients. Medical Sciences.

24. Shen C, Wang Z, Zhao F, (2020) reatment of 5 critically ill patients with COVID-19 with convalescent plasma.JAM Published online March 27.

25. Casadevall A, Pirofski L (2020) The convalescent sera option for containing COVID-19. The Journal of clinical investigation 130(4): 1545-8

26. Arabi YM, Hajeer AH, Luke T, Raviprakash K, Balkhy
H, Johani S, Al-Dawood A, (2016) Feasibility of using convalescent plasma immunotherapy for MERS-CoV infection, Saudi Arabia. Emerging infectious diseases 22(9): 1554.

27. Menis M, Sridhar G, Selvam N, Ovanesov MV, Divan HA, Liang Y, et al. (2013) Hyperimmune globulins and sameday thrombotic adverse events as recorded in a large healthcare database during 2008-2011. Am J Hematol 88: $1035-40$.

28. Marano G, Vaglio S, Pupella S, Facco G, Catalano L, Liumbruno GM, et al. (2016) Convalescent plasma: new evidence for an old therapeutic tool? Blood Transfus 14: $152-7$.

29. Huang C, Wang Y, Li X, (2020) Clinical features of patients infected with 2019 novel corona virus in Wuhan, China. Lancet 395(10223): 497-506. 